Irish Math. Soc. Bulletin

Number 85, Summer 2020, 21-30

ISSN 0791-5578

\title{
A reflection property in Minkowski planes
}

\author{
MOSTAFA GHANDEHARI AND HORST MARTINI
}

\begin{abstract}
With the reflection property investigated here we mean a generalization of the following task, also called Heron's problem: Minimize the length of a path joining two points on one side of a line in the Euclidean plane such that this path meets also this line. We present an extension of this reflection property to (normed or) Minkowski planes and use this generalization to discuss also corresponding reflection properties of Minkowskian conics.
\end{abstract}

\section{INTRODUCTION}

The concept of Minkowski distance defined by means of a convex body centered at the origin was developed by $\mathrm{H}$. Minkowski [21], yielding the notion of Minkowski spaces; these are simply finite dimensional real Banach spaces with the planar subcase of Minkowski planes. The geometry of such spaces and planes is usually called Minkowski geometry, see the monograph [31]. The articles [3] by Busemann, [26] by Petty, the surveys [19] and [18], Chapter 6 from [2] and Chapter 4 from [32] as well as the whole monograph [31] contain useful background material reflecting main directions of Minkowski geometry and also those parts of classical convexity which are needed for it.

In this paper we will deal with the extension of Heron's problem to Minkowski planes and conic sections there. In the Euclidean plane, Heron's problem asks for minimizing the length of a path that joins two points on one side of a line and should meet also this line. Using Fermat's principle of least time and the fact that in a homogeneous medium the time travelled is proportional to the distance travelled one obtains the reflection principle as follows: Consider two points $u, v$ lying in one of the open halfplanes determined by a line $\mathcal{L}$ in the Euclidean plane. A point $w$ on $\mathcal{L}$ such that the Euclidean sum of distances $\|u-w\|_{e}+\|v-w\|_{e}$ is minimum has the property that the reflection of a light ray, say from $u$ to $w$, will pass through $v$. The angle of incidence is equal to the angle of reflection. Our main objective is it to extend this reflection property to normed planes and to apply it to corresponding conics.

A convex body in the Euclidean plane is a compact, convex set having non-empty interior. Any convex body $E$ centered at the origin can be taken to define a Minkowskian distance from $x$ to $y$ by

$$
\|x-y\|=\frac{\|x-y\|_{e}}{r} .
$$

Here $\|x-y\|_{e}$ is the Euclidean distance from $x$ to $y$, and $r$ is the value of the Euclidean radial function of $E$ in the direction of the vector $y-x$. (The Euclidean radial function is the function on $\mathbb{R}^{2}$ whose value at each oint $z$ depends only on the distance of $z$ and the origin $o$.) We will call the standard plane equipped with this new metric a (normed or) Minkowski plane, having $E$ as unit circle.

2020 Mathematics Subject Classification. 52A21, 52A10, 46B20.

Key words and phrases. Conic sections, Heron's problem, Minkowskian conics, Minkowski geometry, normed plane, reflection property.

Received on 30-7-2019; revised 21-4-2020. 


\section{Preliminaries}

Assuming that the unit circle $E$ of a Minkowski plane is smooth and strictly convex, we give in the next section an extension of the reflection principle, and in Section 4 we will discuss the reflection property of conics in Minkowski planes. Note that $E$ is called smooth if through any boundary point of $E$ a unique supporting line passes; and it is called strictly convex if the boundary of $E$ contains no line segments. Consider a Minkowski plane with unit circle E. An E-ellipse is the locus of all points whose sum of Minkowskian distances from two fixed points is constant. An E-hyperbola is the locus of all points whose difference of Minkowskian distances from two fixed points is constant, and an E-parabola is the locus of all points which are equidistant from a given point and a given line. Both $E$-ellipses and $E$-parabolas bound convex regions. Minkowskian analogues of the reflection properties for an ellipse and a hyperbola and the focal property for a parabola are given in Section 4. We refer also to [12], [16], and [14] for various results and properties of Minkowskian analogues of conics.

The following is needed in Section 4. Given a line $\mathcal{L}$ and a point $u \notin \mathcal{L}$, for $v \in \mathcal{L}$ the direction $v-u$ is called transversal to $\mathcal{L}$ provided $\|u-v\|=\min \|u-w\|$ for all $w \in \mathcal{L}$. It is very easy to see that if a homothetic copy of the unit circle $E$ centered at $u$ touches $\mathcal{L}$ at a point $v$, then $u-v$ is transversal to $\mathcal{L}$. (Note that homotheties are transformations with a fixed point $x$ sending each $m \neq x$ to a point $n$ such that $n-s$ is on the same line as $m-s$, but scaled by a real factor $\lambda$.) This natural type of transversality is usually called Birkhoff orthogonality, see the related expository paper [1].

We finish this preliminary part by proving a lemma needed in Section 4 and saying that the sum of distances from $m$ fixed points in a normed space is a convex function.

Lemma 2.1. Consider $m$ points $v_{1}, v_{2}, \ldots, v_{m}$ in a normed space $X$. The function $f$ defined by $f(x)=\sum_{i=1}^{m}\left\|x-v_{i}\right\|$ is convex.

Proof. The statement holds since the norm function is convex, and the sum of convex functions is convex, too.

We remark that the level sets of the function discussed here occur as so-called polyellipses or multifocal ellipses and their higher-dimensional analogues, i.e., as respective generalizations of ellipsoids having $m$ foci (see [14]).

\section{A REFLECTION PROPERTY}

Our main objective in this section is to prove the following theorem giving the Minkowskian analogue of Heron's problem (see Figure 1, where the Euclidean subcase is shown).

Theorem 3.1. Consider two points u, $v$ lying in one of the open halfplanes determined by a line $\mathcal{L}$ in a Minkowski plane with continuously differentiable boundary of the unit circle $E$. A point $p$ on $\mathcal{L}$ such that

$$
\|u-p\|+\|v-p\|=\min _{q \in \mathcal{L}}\{\|u-q\|+\|v-q\|\}
$$

has the following reflection property: Let a homothetic copy $E^{\prime}$ of the unit circle $E$, which is centered at the point $p$, intersect the line segments joining $p$ to $u$ and $v$ in the points $u^{\prime}$ and $v^{\prime}$, respectively. Let $u^{\prime \prime}$ and $v^{\prime \prime}$ be intersections of the tangent lines to $E^{\prime}$ at $u^{\prime}$ and $v^{\prime}$ with $\mathcal{L}$. Then

$$
\left\|p-u^{\prime \prime}\right\|_{e}=\left\|p-v^{\prime \prime}\right\|_{e} \quad\left(\text { and } s o\left\|p-u^{\prime \prime}\right\|=\left\|p-v^{\prime \prime}\right\|\right) .
$$




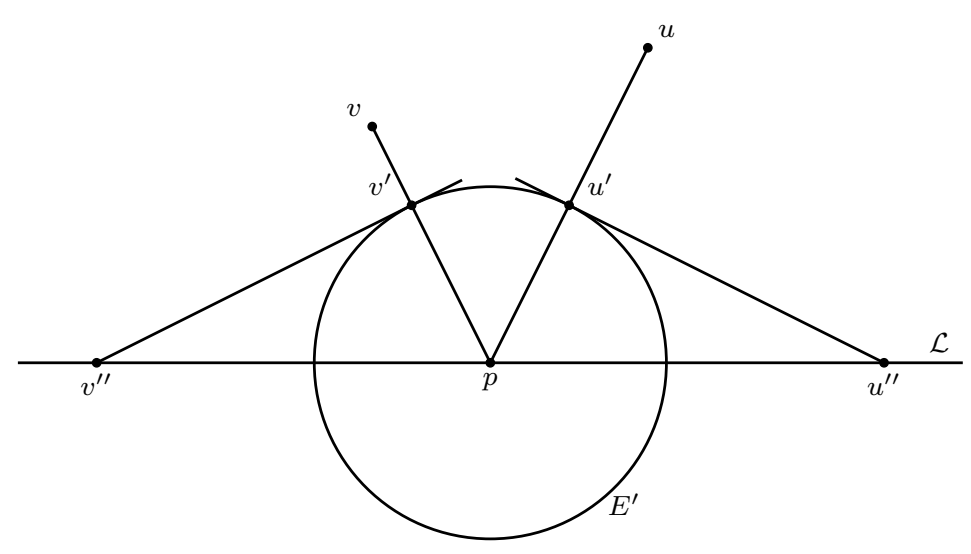

FiguRE 1. E-reflection property

We will use the following Lemma 3.2 (the proof of which is a nice exercise in calculus) to prove Theorem 3.1.

Lemma 3.2. Let $r=g(\vartheta)$ describe a differentiable curve $C$ in polar coordinates. Then the $x$-intercept of the tangent line at a point $(r, \vartheta)$ is given by

$$
x=\frac{r^{2}}{r \cos \vartheta+r^{\prime} \sin \vartheta},
$$

where

$$
r^{\prime}=\frac{d g}{d \vartheta} .
$$

Proof of Theorem 3.1. The function $f(q)=\|q-u\|+\|q-v\|, q \in \mathcal{L}$, is convex and unbounded. Hence $f$ has a minimum on at least one point $p \in \mathcal{L}$. That is, there exists $p \in \mathcal{L}$ with $f(p) \leq f(q)$ for all $q \in \mathcal{L}$. Since the boundary of $E$ is of class $C^{1}$, $f(q)=\|q-u\|+\|q-v\|=F(E, q-u)+F(E, q-v)$ is of class $C^{1}$. Therefore the function $f=\left.f\right|_{\mathcal{L}}$ is of class $C^{1}$, and the method of Lagrange multipliers can be applied.

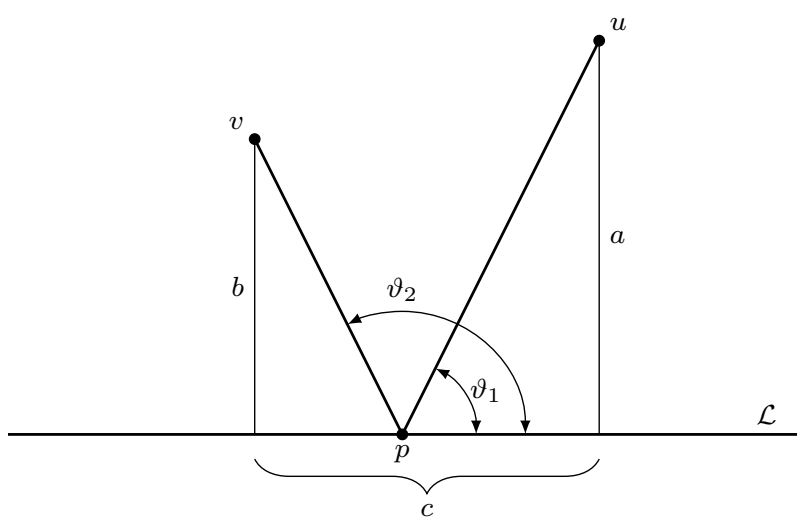

FiguRE 2. For the proof of Theorem 3.1

Using some trigonometry and the definitions of the Minkowski metric, Theorem 3.1 is equivalent to the following constraint optimization problem (see Figure 2):

$$
\text { Minimize } \quad F\left(\vartheta_{1}, \vartheta_{2}\right)=\frac{a \csc \vartheta_{1}}{r\left(\vartheta_{1}\right)}+\frac{b \csc \vartheta_{2}}{r\left(\vartheta_{2}\right)},
$$




$$
\text { subject to } \quad G\left(\vartheta_{1}, \vartheta_{2}\right)=a \cot \vartheta_{1}-b \cot \vartheta_{2}-c=0,
$$

with $a, b, c$ as shown in Figure 2 .

The Lagrangian is denoted by $J$ and given by

$$
J\left(\vartheta_{1}, \vartheta_{2}, \lambda\right)=\frac{a \csc \vartheta_{1}}{r\left(\vartheta_{1}\right)}+\frac{b \csc \vartheta_{2}}{r\left(\vartheta_{2}\right)}-\lambda\left(a \cot \vartheta_{1}-b \cot \vartheta_{2}-c\right) .
$$

Setting $\frac{\partial J}{\partial \vartheta_{1}}, \frac{\partial J}{\partial \vartheta_{2}}$, and $\frac{\partial J}{\partial \lambda}$ equal to zero, we obtain

$$
\begin{gathered}
\frac{\partial J}{\partial \vartheta_{1}}=\frac{-a \csc \vartheta_{1} \cot \vartheta_{1}}{r\left(\vartheta_{1}\right)}-\frac{a \csc \vartheta_{1} r^{\prime}\left(\vartheta_{1}\right)}{r^{2}\left(\vartheta_{1}\right)}+\lambda a \csc ^{2} \vartheta_{1}=0, \\
\frac{\partial J}{\partial \vartheta_{2}}=\frac{-b \csc \vartheta_{2} \cot \vartheta_{2}}{r\left(\vartheta_{2}\right)}-\frac{b \csc \vartheta_{2} r^{\prime}\left(\vartheta_{2}\right)}{r^{2}\left(\vartheta_{2}\right)}-\lambda b \csc ^{2} \vartheta_{2}=0, \\
\frac{\partial J}{\partial \lambda}=-a \cot \vartheta_{1}+b \cot \vartheta_{2}+c=0 .
\end{gathered}
$$

Dividing equations (4) and (5) by $a \csc ^{2} \vartheta_{1}$ and $b \csc ^{2} \vartheta_{2}$, respectively, and using Lemma 3.2 , we obtain

$$
\begin{gathered}
\lambda=\frac{r\left(\vartheta_{1}\right) \cos \vartheta_{1}+r^{\prime}\left(\vartheta_{1}\right) \sin \vartheta_{1}}{r^{2}\left(\vartheta_{1}\right)}=\frac{1}{\left\|p-u^{\prime \prime}\right\|_{e}}, \\
\lambda=-\frac{r\left(\vartheta_{2}\right) \cos \vartheta_{2}+r^{\prime}\left(\vartheta_{2}\right) \sin \vartheta_{2}}{r^{2}\left(\vartheta_{2}\right)}=\frac{1}{\left\|p-v^{\prime \prime}\right\|_{e}},
\end{gathered}
$$

where $u^{\prime \prime}$ and $v^{\prime \prime}$ are the $x$-intercepts of tangent lines to a copy of the unit circle centered at $p$. Points of tangency are intersections of line segments joining $p$ to $u$ and $v$ in the points $u^{\prime}$ and $v^{\prime}$, with the unit circle. Hence $\left\|p-u^{\prime \prime}\right\|_{e}=\left\|p-v^{\prime \prime}\right\|_{e}$, and consequently $\left\|p-u^{\prime \prime}\right\|=\left\|p-v^{\prime \prime}\right\|$.

The reflection property for the Euclidean case is a special case of Theorem 3.1. If $E$ has a vertical axis of symmetry, then the angle of incidence will be equal to the angle of reflection. In the next section we use the above reflection property to discuss the reflection property of conics.

Hawkins [9] used variational techniques to find a generalization of Snell's law of refraction for media, where the speed of light depends only on the direction at each point. He also used the method of Lagrange multipliers to generalize Snell's law, taking a constraint optimization problem similar to that in the proof of Theorem 3.1 into consideration. Ghandehari and Golomb [8] have done similar work.

By methods from convex analysis, Heron's problem was generalized in [22] where the sum of distances to $m$ given closed convex sets is studied. From the numerical point of view, generalizations of Heron's problem are investigated in [5]. An extension to Banach spaces is presented in [23], and we also mention the paper [11] containing related results for Hilbert spaces.

\section{CONICS}

In this section we define conics in the Minkowski plane and analyze their reflection properties. The excellent book by Hilbert and Cohn-Vossen has a good introduction to conics in the Euclidean plane, and for Minkowskian conics we refer to [12] and [16].

Suppose $E$ is a smooth, convex and compact body in the plane inducing a Minkowski norm $\|\cdot\|$. If $u$ and $v$ are distinct points and $\mathcal{L}$ is a line, we say that a point $p \in \mathcal{L}$ has the $E$-reflection property with respect to $u, v$, and $\mathcal{L}$ if $\|u-p\|+\|v-p\|=\min _{z \in \mathcal{L}}\{\| u-$ $z\|+\| v-z \|\}$.

In the Euclidean plane, the line segments joining the two foci to a point on an ellipse will make equal angles with the tangent line to the ellipse at the point of tangency. This is called the reflection property of an ellipse. A short and elegant proof of the reflection 
property of an ellipse using derivatives is given in Schulz and Moore [28]. Recall that we defined an $E$-ellipse in a Minkowski plane with unit circle $E$ as the locus of all points whose Minkowskian sum of distances from two fixed points is constant. We shall not use the definition of an ellipse by using eccentricity here, but mention that Tamássy and Bélteky [30] showed that if in a Minkowski plane the characterization of an ellipse in terms of sums of distances coincides with that via eccentricity, then the plane is Euclidean.

The following Theorem 4.2 concerns the Minkowskian analogue of the reflection property of an E-ellipse. The proof is the same as in the Euclidean case. Before stating and proving Theorem 4.2, we need the following lemma.

Lemma 4.1. The region bounded by an E-ellipse is convex.

Proof. Let $D=\left\{x \in \mathbb{R}^{2} \mid\|x-u\|+\|x-v\| \leq d\right\}$, where $u$ and $v$ are the foci and $d$ is the constant sum of distances of points of the $E$-ellipse from $u$ and $v$. Let $f(x)=$ $\|x-u\|+\|x-v\|$. By Lemma 2.1 we have that if $x \in D, y \in D$, then, for $0 \leq \lambda \leq 1$,

$$
f(\lambda x+(1-\lambda) y) \leq \lambda f(x)+(1-\lambda) f(y) \leq \lambda d+(1-\lambda) d=d .
$$

Hence $D$ is convex.

We note that the unit circle $E$ is strictly convex if and only if $E$-ellipses are strictly convex (see [12]).

Theorem 4.2. Let $u$ and $v$ be foci of an E-ellipse in a Minkowski plane with smooth and strictly convex unit circle E. Let $\mathcal{L}$ be the tangent line to the E-ellipse at a point $p$. Then $p$ has the E-reflection property with respect to $u, v$, and $\mathcal{L}$ (see Figure 3 for the Euclidean subcase).

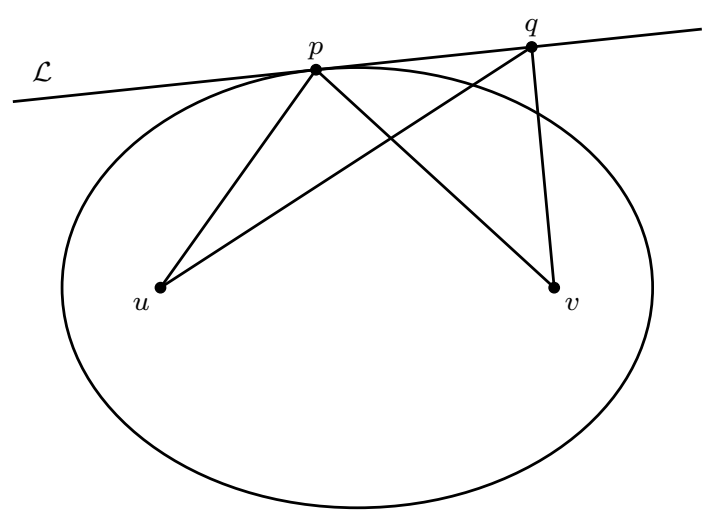

Figure 3. Reflection property of an E-ellipse

Proof. Since the $E$-ellipse is a convex curve, any point $q \in \mathcal{L}, q \neq p$, lies outside of the $E$-ellipse. Then $\|q-u\|+\|q-v\| \geq\|p-u\|+\|p-v\|$. Hence $p$ has the $E$-reflection property.

In what follows, Theorem 4.3 deals with a property of a point $p$ on the unit circle such that $\|p-u\|+\|p-v\|$ is a minimum for given points $u, v$ in a Minkowski plane. Theorem 4.4 is based on Theorem 4.3 and gives a property of the Fermat-Torricelli point for three given points $u, v$, and $w$ (i.e., of the unique point having minimal sum of distances to $u, v$, and $w$ ). Theorem 4.5 is a generalization of Theorem 3.1 where, given a point $u$ and two lines $\mathcal{L}_{1}$ and $\mathcal{L}_{2}$, we find points $p \in \mathcal{L}_{1}$ and $v \in \mathcal{L}_{2}$ such that 
$\|p-u\|+\|p-v\|$ is minimum. We then use Theorem 4.5 to prove Theorem 4.7, which is the focal property for an $E$-parabola. Theorem 4.8 gives the reflection property for an $E$-hyperbola, and we finish the article with a conjecture referring to confocal ellipses.

Theorem 4.3. Consider two points $u$ and $v$ such that the line segment connecting $u$ and $v$ lies outside of a homothetic copy of the strictly convex unit circle $E$. A point $p$ on the copy of $E$ minimizes $\|u-p\|+\|v-p\|$ if and only if $p$ has the E-reflection property with respect to $u, v$, and the line $\mathcal{L}$ tangent to the copy of $E$ at $p$ (see Figure 4).

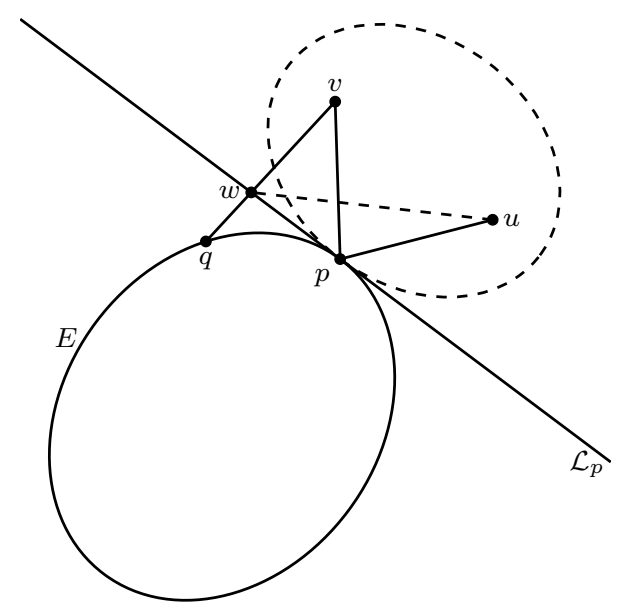

FiguRE 4. $p$ minimizes $\|p-u\|+\|p-v\|$

Proof. We first prove that if $p \in E$ such that $\|u-p\|+\|v-p\|=\min _{z \in \mathcal{L}_{p}}\{\|u-z\|+\|v-z\|\}$ with $\mathcal{L}_{p}$ tangent to $E$ at $p$, then $\|u-p\|+\|v-p\|=\min _{q \in E}\{\|u-q\|+\|v-q\|\}$. For any $q \in E$, let $w$ be the intersection of the line segment, connecting $q$ and $v$ outside of $E$, with the tangent line $\mathcal{L}_{p}$ at $p$ (see again Figure 4). Then $\|u-q\|+\|q-v\|=$ $\|u-q\|+\|q-w\|+\|w-v\|$, since $\mathcal{L}_{p}$ meets the line segment $[q, v]$. This follows from the assumption on $p$ formulated at the beginning of the proof. Further on, we have $\|u-q\|+\|q-w\|+\|w-v\| \geq\|u-w\|+\|w-v\| \geq\|u-p\|+\|v-p\|$, where the last inequality follows since $w \in \mathcal{L}_{p}$ and $\|p-u\|+\|v-p\|=\min _{z \in \mathcal{L}_{p}}\{\|z-u\|+\|v-z\|\}$.

Hence, if $p$ has the $E$-reflection property with respect to $u, v$, and the tangent line $\mathcal{L}_{p}$ at $p$, then $\|u-p\|+\|v-p\|=\min _{q \in E}\{\|u-q\|+\|v-q\|\}$.

If $\|u-p\|+\|v-p\|=\min _{q \in E}\{\|u-q\|+\|v-q\|\}$, then $\|u-p\|+\|v-p\|=\min _{z \in \mathcal{L}_{p}}\{\| u-$ $z\|+\| v-z \|\}$ as follows: The $E$-ellipse, with foci $u$ and $v$ and the constant sum of distances equal to the minimum, intersects $E$ at only one point. If there are two points of intersection, then for any $x$ inside the line segment joining two points of intersection, $x$ is inside $E$ since $E$ is strictly convex. Then $\|u-x\|+\|v-x\| \leq\|u-p\|+\|v-p\|$ since $E$ is strictly convex. Let $y$ be some chosen point from the intersection of the line segment joining $x$ to $v$ with $E$. Then $\|u-x\|+\|v-x\|=\|u-x\|+\|x-y\|+\|y-v\|>$ $\|u-y\|+\|u-v\| \geq\|u-p\|+\|v-p\|$, a contradiction. Hence there is only one point $p$ of intersection. The tangent line to $E$ at $p$ is also tangent to the $E$-ellipse. By Theorem $4.2, p$ has the $E$-reflection property with respect to $u, v$, and the tangent line $\mathcal{L}_{p}$.

A problem related to the reflection property is the so-called Fermat-Torricelli problem. In its simplest form, it asks for a point in the Euclidean plane minimizing the sum 
of the distances to three given points. In the article [4] the Fermat-Torricelli problem in Minkowski spaces is investigated, see also [20].

Theorem 4.4. Consider three points $u, v$, and $w$ in a given Minkowski plane with smooth and strictly convex unit circle E. Let $p$ be the Fermat-Torricelli point, assuming it exists and is different from $u, v$, or $w$. Let $u^{\prime}, v^{\prime}$ and $w^{\prime}$ be the intersections of a copy of $E$ centered at $p$ with the line segments joining $p$ to $u, v$, , and $w$. Then the triangle formed by drawing tangents to $E$ at $u^{\prime}, v^{\prime}$, and $w^{\prime}$ has $p$ as its centroid.

Proof. Consider a copy of the unit circle $E$ centered at $w$ and passing through $p$. Since $\|u-p\|+\|v-p\|$ is minimized as $p$ varies on this copy of the unit circle, $p$ has the $E$-reflection property with respect to $u, v$, and the tangent line $\mathcal{L}_{1}$ to this copy of the unit circle. We now consider another copy of the unit circle, centered at $p$ and passing through $w$. Let $u^{\prime}$ and $v^{\prime}$ be the intersection with line segments joining $p$ to $u$ and $v$, respectively. Let $\mathcal{L}_{2}$ be a line tangent to a copy of the unit circle at $w$ with $\mathcal{L}_{2} \| \mathcal{L}_{1}$. By the E-reflection property (Theorem 3.1), the line segment passing through $p$ and parallel to $\mathcal{L}_{2}$ is bisected by the sides of the triangle formed by the tangents. Using the same argument for $u$ and $v$ we see that the line segment passing through $p$ and being parallel to $\mathcal{L}_{2}$ is bisected by the sides of the triangle formed by tangents. Using the same argument for $u$ and $v$ we see that the line segments passing through $p$ and parallel to the sides of the triangle formed by the tangents are bisected. It is an elementary but interesting exercise to show that the point $p$ has to be the centroid.

The excellent book by Courant and Robbins [6] has a treatment of extremal distances in the Euclidean plane. Similar results for Minkowski planes can be obtained. We will use the following theorem to discuss the focal property of parabolas.

Theorem 4.5. Consider a point $u$ and two lines $\mathcal{L}_{1}$ and $\mathcal{L}_{2}$ in a Minkowski plane with smooth and strictly convex unit circle $E$. If $\|v-p\|+\|u-p\|$ is a minimum for $p \in \mathcal{L}_{1}$ and $v \in \mathcal{L}_{2}$, then $p$ has the E-reflection property with respect to $u, v$, and $\mathcal{L}_{1}$.

Proof. If $p \in \mathcal{L}_{1}$ and $v \in \mathcal{L}_{2}$ attain this minimum, then the minimum of $\|u-z\|+\|v-z\|$ as $z$ runs along $\mathcal{L}_{1}$ is attained at $z=p$.

Hence $p$ has the $E$-reflection property with respect to $u, v$, and $\mathcal{L}_{1}$.

For a parabola in the Euclidean plane, the path of reflection of a light ray starting from the focus and going to the boundary is called its focal property. Theorem 4.7 below gives an analogue of this focal property in the Minkowski plane. Before this, we show that the region bounded by an $E$-parabola is convex.

Lemma 4.6. An E-parabola bounds a convex region.

Proof. Let $\mathcal{L}$ and $u$ be the line and the point generating the $E$-parabola in the classical way. Let $D=\{x \mid\|x-u\| \leq \rho(x, \mathcal{L})\}$ where $\rho(x, \mathcal{L})$ is the distance of $x$ from $\mathcal{L}$. If $x, y \in D$ and $0 \leq \lambda \leq 1$, then $\|\lambda x+(1-\lambda) y-u\| \leq \lambda\|x-u\|+(1-\lambda)\|y-u\| \leq$ $\lambda \rho(x, \mathcal{L})+(1-\lambda) \rho(y, \mathcal{L})=\rho(\lambda x+(1-\lambda) y, \mathcal{L})$. The last equality follows from the fact that transversal directions to $\mathcal{L}$ are all parallel.

Laatsch [17] gave an interesting treatment of pyramidal sections in taxicab geometry which is a special case of Minkowski geometry, with unit circle $E$ a square centered at the origin and diagonals on the $x$ - and $y$-axes. For other references on conics in Taxicab geometry see the articles by Iny [13], Moser and Kramer [24], Reynolds [27], and Sowell [29]. For a computerized approach to conics with Taxicab metric we refer to Natsoulas $[25]$.

The following theorem gives the focal property of a Minkowskian parabola, which is generated as locus of all points equidistant from a given point (focus) and a given line 
(directrix). For a short geometric proof of the focal property of the parabola in the Euclidean plane, see Williams [33].

Theorem 4.7. (Focal property). For an E-parabola with focus $u$ and directrix $\mathcal{L}$, let $\mathcal{L}^{\prime}$ be any line parallel to $\mathcal{L}$ such that $u$ is between $\mathcal{L}$ and $\mathcal{L}^{\prime}$. For any point $p$ on the $E$ parabola, choose $v \in \mathcal{L}^{\prime}$ such that $p-v$ is transversal to $\mathcal{L}^{\prime}$. Then $p$ has the E-reflection property with respect to $u, v$, and the tangent line to the E-parabola at $p$.

Proof. Let $\mathcal{L}^{\prime \prime}$ be the tangent line to the $E$-parabola at $p$. For $q \in \mathcal{L}^{\prime \prime}$, choose $w \in \mathcal{L}^{\prime}$ such that $q-w$ is transversal to $\mathcal{L}^{\prime}$. Let $q^{\prime}$ be the intersection of the line segment joining $q$ to $u$ with the $E$-parabola. Choose $w^{\prime}$ on $\mathcal{L}^{\prime}$ such that $q^{\prime}-w^{\prime}$ is transversal to $\mathcal{L}^{\prime}$. Then

$$
\begin{aligned}
& \|u-q\|+\|q-v\| \geq\|u-q\|+\|q-w\| \geq\left\|u-q^{\prime}\right\|+\left\|q^{\prime}-w\right\| \geq \\
& \left\|u-q^{\prime}\right\|+\left\|q^{\prime}-w^{\prime}\right\|=\|u-p\|+\|p-v\|,
\end{aligned}
$$

where the first inequality follows from the triangle inequality for the points $q^{\prime}, q, w$, and the second inequality follows since $q^{\prime} w^{\prime}$ is transversal to $\mathcal{L}^{\prime}$. The last equality holds since the points $p$ and $q^{\prime}$ both lie on the $E$-parabola. Thus, $p$ has the $E$-reflection property with respect to $u, v$, and $\mathcal{L}^{\prime \prime}$.

The following theorem gives the reflection property of an E-hyperbola. Chapter 3 of the book by Kazarinoff [15] contains a good treatment of reflection properties in the Euclidean plane. In particular, a proof of the reflection property for a hyperbola is given there. The book by Courant and Robbins [6] contains a treatment of extremal distances in the Euclidean plane and a nice discussion of Heron's reflection principle and the reflection property of conics.

Theorem 4.8. Let $u$ and $v$ be two foci of an E-hyperbola in a Minkowski plane with smooth and strictly convex unit circle E. Let $p$ be a point on a branch of the hyperbola containing the focus $v$. Assume $v^{\prime}$ is the reflection of $v$ through $p$. Consider a line $\mathcal{L}$ through $p$ such that $p$ has the E-reflection property with respect to $u, v^{\prime}$, and $\mathcal{L}$. Then $\mathcal{L}$ is tangent to the hyperbola at $p$ (see Figure 5 for the Euclidean subcase).

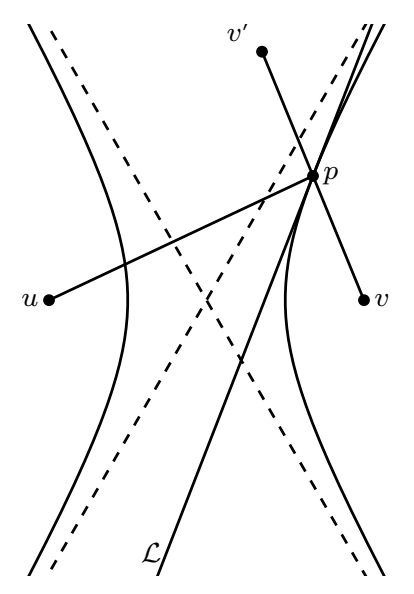

FIgURE 5. The tangent property of the hyperbola

Proof. Suppose the line $\mathcal{L}$ intersects the hyperbola in another point $q$. Then

$$
\begin{aligned}
& \|u-q\|+\left\|q-v^{\prime}\right\|=\|u-q\|-\|v-q\|+\|v-q\|+\left\|q-v^{\prime}\right\| \\
= & \|p-u\|-\|p-v\|+\|v-q\|+\left\|q-v^{\prime}\right\| \\
> & \|p-u\|-\|p-v\|+2\|p-v\| \\
= & \|p-u\|+\|p-v\|,
\end{aligned}
$$


where we have used the definition of an $E$-hyperbola and the triangle inequality for the triangle $v q v^{\prime}$. Thus

$$
\|u-q\|+\left\|q-v^{\prime}\right\|>\|p-u\|+\|p-v\|,
$$

giving a contradiction to the fact that $p$ has the $E$-reflection property with respect to $u, v^{\prime}$ and $\mathcal{L}$. Hence $\mathcal{L}$ is tangent to the hyperbola.

We conclude the paper by a conjecture which would generalize the following result on Euclidean reflections for confocal ellipses (see Figure 6): Consider two confocal ellipses with foci $f_{1}$ and $f_{2}$. Assume that $p$ is a point on the larger ellipse. Draw the tangent line $\mathcal{L}$ to the larger ellipse at $p$. From $p$ draw two tangents to the smaller ellipse, with $a$ and $b$ as points of tangency, respectively. Then $\overline{a p}$ and $\overline{b p}$ have the reflection property with respect to $\mathcal{L}$. That is, in Figure 6 the angles $\alpha$ and $\beta$ are equal.

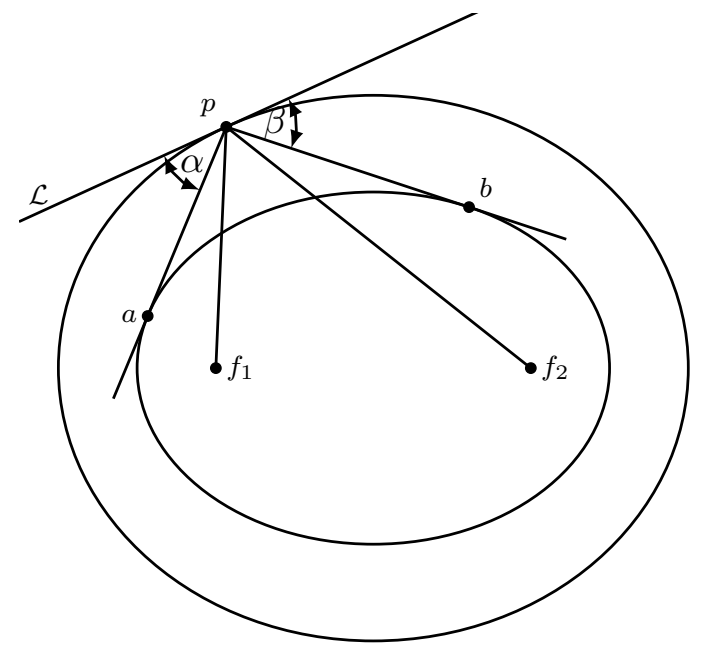

Figure 6. Confocal ellipses

Conjecture. Consider an analogous construction of confocal ellipses in a Minkowski plane with smooth unit circle. Then the Minkowskian reflection property holds between $\overline{a p}, \overline{b p}$, and $\mathcal{L}$.

\section{REFERENCES}

[1] J. Alonso, H. Martini, S. Wu: On Birkhoff orthogonality and isosceles orthogonality in normed linear spaces. Aequationes Math. 83 (2012), no. 1-2, 153-189.

[2] R. V. Benson: Euclidean Geometry and Convexity. McGraw-Hill Book Co., New York, 1966.

[3] H. Busemann: The foundations of Minkowski geometry. Comment. Math. Helvet. 24 (1950), 156187.

[4] G. D. Chakerian, M. A. Ghandehari: The Fermat problem in Minkowski spaces. Geom. Dedicata 17 (1985), no. 3, 227-238.

[5] E. Chi, K. Lange: A look at the generalized Heron problem through the lens of majorizationminimization. Amer. Math. Monthly 121 (2014), no. 2, 95-108.

[6] R. Courant, H. Robbins: What is Mathematics? Oxford University Press, Sixth Printing, New York, 1953. (pp. 329-343)

[7] M. Gardner: Mathematical games. Scientific American 243, No. 2 (Nov. 1980), 18-30.

[8] M. Ghandehari, M. Golomb: Minimum path problems in normed spaces: reflection and refraction. J. Optim. Theory Appl. 105 (2000), 1-16.

[9] M. Hawkins: A Generalization of Snell's Law. Master's Thesis, Naval Postgraduate School, 1990.

[10] D. Hilbert, S. Cohn-Vossen: Geometry and the Imagination. Translated by P. Neményi, Chelsea Publishing Company, New York, 1952. (pp. 1-11) 
[11] F. Holland, A. Mustata: A Hilbert space analogue of Heron's reflection principle. Irish Math. Soc. Bull. 67 (2011), 57-65.

[12] Á G. Horváth, H. Martini: Conics in normed planes. Extracta Math. 26 (2011), no. 1, 29-43.

[13] D. Iny: Taxicab geometry: another look at conic sections. Pi Mu Epsilon J. 7 (1984), 645-647.

[14] Th. Jahn, H. Martini, C. Richter: Bi- and multifocal curves and surfaces for gauges. J. Convex Anal. 23 (2016), no. 3, 733-774.

[15] N. D. Kazarinoff: Geometric Inequalities. New Mathematical Library, 4 Random House, New York-Toronto, 1961.

[16] Á. Kurusa: Conics in Minkowski geometries. Aequationes Math. 92 (2018), 949-961.

[17] R. Laatsch: Pyramidal sections in taxicab geometry. Math. Mag. 55 (1982), no. 4, 205-212.

[18] H. Martini, K. J. Swanepoel: The geometry of Minkowski spaces - a survey, Part II. Expositiones Math. 22 (2004), 93-144.

[19] H. Martini, K. J. Swanepoel, G. Weiss: The geometry of Minkowski spaces - a survey, Part I. Expositiones Math. 19 (2001), no. 2, 97-142.

[20] H. Martini, K. J. Swanepoel, G. Weiss: The Fermat-Torricelli problem in normed planes and spaces. J. Optim. Theory Appl. 115 (2002), 283-314.

[21] H. Minkowski: Theorie der konvexen Körper, insbesondere Begründung ihres Oberflächenbegriffs. In: Ges. Abh., Vol. 2, pp. 131-229, Leipzig und Berlin, 1911.

[22] B. Mordukhovich, Ng. M. Nam, J. Salinas: Solving a generalized Heron problem by means of convex analysis. Amer. Math. Monthly 119 (2012), no. 2, 87-99.

[23] B. Mordukhovich, Ng. M. Nam, J. Salinas: Applications of variational analysis to a generalized Heron problem. Appl. Anal. 91 (2012), no. 10, 1915-1945.

[24] J. Moser, F. Kramer: Lines and parabolas in Taxicab geometry. Pi Mu Epsilon J. 7 (1982), 441-448.

[25] A. Natsoulas: Taxicab conics: An exploration into the world of Taxicab geometry. J. Comput. Math. Sci. Teaching, Summer 1989, pp. 39-47.

[26] C. M. Petty: On the geometry of the Minkowski plane. Riv. Mat. Univ. Parma 6 (1955), 269-292.

[27] B. E. Reynolds: Taxicab geometry. Pi Mu Epsilon J. 7 (1982), 77-88.

[28] W. C. Schulz, C. G. Moore: Reflections on the ellipse. Math. Mag. 60 (1987), no. 3, 167-168.

[29] K. O. Sowell: Taxicab geometry - a net slant. Math. Mag. 62 (1989), 238-248.

[30] L. Tamássy, K. Bélteky: On the coincidence of two kinds of ellipses in Minkowskian spaces and in Finsler planes. Publ. Math. Debrecen 31 (1984), no. 3-4, 157-161.

[31] A. C. Thompson: Minkowski Geometry. Cambridge University Press, Cambridge, 1996.

[32] F. A. Valentine: Convex Sets. Krieger, New York, 1976.

[33] R. C. Williams: A proof of the reflection property of the parabola. Amer. Math. Monthly 94, no. 7, 667-668.

Mostafa Ghandehari has been distinguished senior lecturer of civil engineering and adjunct professor of mathematics. He has taught mathematics at various levels, and also computer science at UT Arlington as well as Northern California.

Horst Martini was a teacher of mathematics, geography, and astronomy. He later specialized in several mathematical fields, such as discrete and convex geometry, Banach-space theory and combinatorics. He taught mathematics as full professor at the University of Technology in Chemnitz, Germany.

(M. Ghandehari) Dept. of Mathematics, University of Texas at Arlington, Arlington, TX 76019 , U.S.A.

(H. Martini) Horst Martini, Fakultät für Mathematik, Technische Universität Chemnitz, GERMANy

E-mail address, M. Ghandehari: ghandeha@uta.edu

E-mail address, H. Martini: martini@mathematik.tu-chemnitz.de 